CONTRIBUTION OF SOVIET-BOLIVIAN ASTRONOMICAL OBSERVATORY IN THE CONSTRUCTION OF THE INERTIAL COORDINATE SYSTEM

\author{
D.D. Polozhentsev, H.I. PotTer and L.I. YaGudin \\ Central Astronomical Observatory \\ Pulkovo \\ 196140 Leningrad, USSR \\ and \\ J.A. Zelaya and R.F. Zalles \\ Observ. Astron. Nacional \\ Tarija, Bolivia
}

ABSTRACT. The work on photographic astrometry in Bolivia begun in 1983, when an expeditional astrograph was put there $\left(D=23 \mathrm{~cm}, F=230 \mathrm{~cm}\right.$, working field of $\left.4^{\circ} \times 4^{\circ}\right)$. The main purpose of the observations with this telescope is extending the inertial coordinate system to different classes of stars. For this purpose we have carried out and are currently carrying out the following observational astrometric programs:

1. The preliminary catalog of precise positions of 200000 southern stars to mag 11 with declination in the range from $0^{\circ}$ to $-90^{\circ}$ was created. Each star was observed four times with displacement of the photoplate by $2^{\circ}$ in $\delta$ and by $8 \sec \delta$ in $\alpha$. The exposure time is from 4 to 12 minutes. The accuracy of the preliminary catalog is \pm 0.247 arcsec and the final one is \pm 0.15 arcsec.

2. We are not far from finishing the work on a catalog of 1900 bright $\operatorname{stars}\left(-90^{\circ}<\delta ; 0^{\circ}, \mathrm{m} \leq 6.05\right)$. Each star was observed not less than 2 times for different positions of a special filter which diminished the light of bright stars.

3. The work on astrometry of equatorial stars to mag 12 in the range of declination from $-20^{\circ}$ to $+20^{\circ}$ was begun. From the beginning of the observations on 1 May 1987, 1150 astroplates were obtained. On the whole it is necessary to obtain 3840 photoplates. All three programs use the reference catalog SRS, compiled by Pulkovo and Washington astronomers, in the FK5 system.

\title{
THE PROBLEM OF THE LEVELING OF THE RESERVED NET OF ARCS AT THE CELESTIAL SPHERE BY THE CONSTRUCTION OF THE REFERENCE COORDINATE SYSTEM
}

\author{
E.I. YAGUdina \\ Institute of Applied Astronomy \\ 8 Zhdanovskaya ul. \\ 197042 Leningrad, USSR
}

ABSTRACT. The methods of the rational leveling of the results of the observations of the angular distances between quasars by VLBI method were suggested. The application of these methods on the model of reserved net on sphere shows a descrease of the standard error of the quasers' coordinates after leveling by a factor of three times. We employed 10 elementary configurations constructed on 20 chosen quasars in the Northern hemisphere. 